\title{
Albendazole in the treatment of pulmonary echinococcosis
}

\author{
Praveen Aggarwal, Jyoti Prakash Wali
}

\begin{abstract}
Ten patients with pulmonary hydatid disease diagnosed on the basis of a chest radiograph and a positive response to the indirect haemagglutination test for hydatid disease were treated with albendazole $10 \mathrm{mg} / \mathrm{kg} / \mathrm{day}$ for eight weeks. None of the 10 patients showed any radiological or serological improvement with this treatment regimen. Albendazole in these doses appears to have little role in the treatment of pulmonary hydatid disease.
\end{abstract}

Until recently the treatment of hydatid disease usually required surgical removal of the cysts. In the last decade benzimidazole drugs have been used to treat hydatid disease in man but the results have been variable. Most of the trials have been conducted on patients with hydatid disease of the liver. Albendazole, which has a better absorption than mebendazole, has produced encouraging early results. ${ }^{1}$ This prompted us to study the efficacy of albendazole in the treatment of hydatid disease of the lung in Indian patients.

\section{Methods}

From January 1986 to September 1989 we saw 13 patients at our hospital who were suspected of having pulmonary hydatid disease on the basis of a chest radiograph. In 10 of the patients the serological test for hydatid disease using an indirect haemagglutination assay gave a positive result (titre $\geqslant 1: 128$ ); these patients were included in the study. Their age ranged from 17 to 40 years and their weight from 55 to $62 \mathrm{~kg}$ (mean weight $58 \cdot 1$ $\mathrm{kg}$ ). All had had cough and scanty expectoration for 4-12 months. Five patients had had slight haemoptysis and four had dull chest pain. Seven patients underwent computed tomography of the chest, which showed daughter cysts inside the parent cyst. Three of the 10 patients had multiple cysts.

The patients were treated with albendazole (Alminth, Torrent Laboratories Ltd, Ahmadabad) $10 \mathrm{mg} / \mathrm{kg}$ daily in three divided doses after food for eight weeks. In the hope of increasing compliance, patients were asked to purchase the drug each week. Compliance was checked by verbal questioning and by examining the medicine bills each week. Informed consent was obtained from each patient.

The baseline full blood count, serum ala- nine aminotransferase and aspartate aminotransferase activity, and bilirubin concentration were determined before treatment was started. These tests were repeated weekly for eight weeks. At the end of eight weeks a chest radiograph and serological test for hydatid disease were repeated.

\section{Results}

Patients' compliance was estimated to be over $95 \%$. The initial antibody titres as determined by the indirect haemagglutination test were $1 / 128(n=3), 1 / 256(n=2), 1: 512(n=3)$, and titre $1 / 1024(n=2)$. After completion of eight weeks' treatment only one patient showed symptomatic improvement and this was slight (disappearance of chest pain, but the cough persisted). In the other nine cases the symptoms persisted. None of the patients showed any radiological improvement. There was no change in antibody titre in any patient. No side effects were noted and in particular no fever, hair loss, dizziness, nausea, vomiting, or epigastric pain. The full blood count and results of liver function tests were normal throughout the treatment.

To prevent the complications of rupture of the cysts and superinfection, cysts were removed surgically in seven patients after failure of medical treatment. Histopathological examination showed an intact germinal layer along with daughter cysts in all seven. These seven patients were doing well at follow up after three to 12 months. The other three patients have been followed up for six to 14 months; all continue to have symptoms but with no increase in the size of the cysts.

\section{Discussion}

This open study shows that treatment of hydatid cyst in the lung with albendazole 10 $\mathrm{mg} / \mathrm{kg} /$ day for eight weeks is ineffective. This lack of response to albendazole is in sharp contrast to the results of some earlier studies. Morris et al reported partial response in two patients with lung hydatid cysts treated with albendazole $10 \mathrm{mg} / \mathrm{kg} /$ day for three months. ${ }^{2}$ One patient showed a pronounced reduction in the size of all the cysts after two months' albendazole at a dose of $10 \mathrm{mg} / \mathrm{kg} / \mathrm{day}^{3}$ and another, treated with mebendazole $200 \mathrm{mg}$ /day for three months, showed complete resolution of the cyst. ${ }^{4}$ Okelo ${ }^{5}$ gave albendazole $10 \mathrm{mg} / \mathrm{kg}$ / day to three patients with pulmonary hydatid disease and found substantial improvement in two after treatment for two months and a partial response in the third patient after two 
months' treatment, with a complete response after treatment for a further month with an increased dose of $15 \mathrm{mg} / \mathrm{kg} /$ day. In another report three of four patients with lung hydatid cysts responded to abendazole $12.5 \mathrm{mg} / \mathrm{kg} /$ day. ${ }^{6}$ Of five patients studied by Todovov et $a l,{ }^{7}$ however, three showed only a partial response and two failed to respond. Horton, ${ }^{1}$ reviewing the combined experience of the use of albendazole to treat pulmonary hydatid cysts, noted that most patients had received $800 \mathrm{mg} /$ day of albendazole in cycles of 28 days with a 14 days' interval and a mean of 2.5 cycles. Of the 86 patients with lung hydatid cysts (either alone or with other organs affected) $40 \%$ of the patients were cured and $35 \%$ showed improvement after albendazole treatment.

The dosage regimens for albendazole for the treatment of hydatid diseases have not been standardised; we used a dose of $10 \mathrm{mg} / \mathrm{kg} /$ day for two months. Most previous reports published in English have used the same dose for one to three months for lung or liver hydatid disease. ${ }^{89}$ Most of the cysticidal activity of albendazole appears to occur within the first two to three months of treatment, and if the cyst is not smaller after treatment for two months further drug treatment is unlikely to help. ${ }^{9}$

Side effects of albendazole include gastrointestinal side effects, alopecia, rash, leucopenia, and raised liver enzyme activities. In the review by Horton ${ }^{1} 4 \%$ of patients given albendazole had haematological side effects and $17 \%$ had raised liver enzyme activities. None of these side effects was noted in our patients.

Serological results of medical treatment for hydatid disease have been reported in only a few studies. In one study, in which complement fixation tests gave positive results initially in three of four cases, two patients showed a fourfold rise in antibody titre with successful treatment. ${ }^{3}$ In a study of nine patients antibody titres increased at least fourfold in all but one case, the increase occurring during or within three months of the end of treatment with albendazole. ${ }^{10}$ An initial fall in serological titres did not predict clinical improvement. In the present study there was no change in antibody titres with treatment.

Surgery is the treatment of choice for hydatid disease of the lung unless there is a contraindication. A spontaneous cure occurs occasionally through rupture of the cyst into a bronchus, but the dangers of bronchial spread are too great to justify a waiting policy. ${ }^{11}$

Thus in our small open study albendazole at a dose of $10 \mathrm{mg} / \mathrm{kg} /$ day for eight weeks was ineffective in the treatment of hydatid disease of the lung. The poor outcome, by comparison with some previous studies, could be due to differences in the pharmacokinetics of albendazole in our population or in the susceptibility of the cysts in the small numbers of patients in the different studies. Medical treatment should be considered possibly for patients in whom the cyst is inoperable because of dissemination or a difficult location or because surgery is contraindicated.

1 Horton RJ. Chemotherapy of Echinococcus infection in man with albendazole. Trans $R$ Soc Trop Med Hyg 1989;83: 97-102.

2 Morris DL, Dykes PW, Marriner S. Albendazole-Objective evidence of response in human hydatid disease. JAMA 1983;253:2053-7.

3 Morris DL, Dykes PW, Dickson B, Marriner SE, Bogan JA, Burrows PG. Albendazole in hydatid disease. $B M J$ 1983;286:103-4.

4 Shivashankar A, Lature LH, Shankar PS. Treatment of pulmonary hydatid cyst with mebendazole. $J$ Ass Physicians India 1982;30:317-8.

5 Okelo GB. Hydatid disease: Research and control in Turkans. III. Albendazole in the treatment of inoperable hydatid disease in Kenya-a report on 12 cases. Trans $R$ Soc Trop Med Hyg 1986;80:193-5.

6 Davis A, Pawlowski ZS, Dixon H. Multicentric clinical trials of benzimidazolecarbamates in human echinococcosis. Bull WHO 1986;64:383-8.

7 Todorov V, Vutova K, Petkov D, Mechkov G, Kolev K. Albendazole treatment of human cystic echinococcosis. Trans $R$ Soc Trop Med Hyg 1988;82:453-9.

8 Steiger U, Cotting J, Reichen J. Albendazole treatment of ism and drug tolerance. Clin Pharmacol Ther 1990; ism and drug

9 Gossetto D, Grueneswald S, Antico V, Little JM. Albendazole treatment of recurrent hydatid disease: serial evaluation with ultrasound. Aust NZ J Surg 1989;59: 933-6.

10 Wilcox MH, Morris DL, Bailey JW. Serology in patients treated with albendazole for hydatid disease. $J R$ Soc Med treated with alben

11 Xanthakis D, Efthimiadis M, Papadakis G, et al. Hydatid disease of the chest. Report of 91 patients surgically treated. Thorax 1972;27:517-28. 